\title{
Spinon Fermi Surface Spin Liquid in a Triangular Lattice Antiferromagnet $\mathrm{NaYbSe}_{2}$
}

Peng-Ling Dai, ${ }^{1, \|}$ Gaoning Zhang, ${ }^{2, \|}$ Yaofeng Xie, ${ }^{3}$ Chunruo Duan, ${ }^{3}$ Yonghao Gao, ${ }^{4}$ Zihao Zhu, ${ }^{4}$ Erxi Feng, ${ }^{5}$ Zhen Tao, ${ }^{1}$ Chien-Lung Huang, ${ }^{3}$ Huibo Cao $\odot,^{5}$ Andrey Podlesnyak $\odot,{ }^{5}$ Garrett E. Granroth $\odot,{ }^{5}$ Michelle S. Everett $\odot,{ }^{5}$ Joerg C. Neuefeind $\odot,{ }^{5}$ David Voneshen, ${ }^{6,7}$ Shun Wang, ${ }^{8}$ Guotai Tan $\odot,{ }^{1}$ Emilia Morosan, ${ }^{3}$ Xia Wang, ${ }^{2}$ Hai-Qing Lin, ${ }^{9}$ Lei Shu,${ }^{4}$ Gang Chen, ${ }^{10,4, *}$ Yanfeng Guo $\odot^{2, \dagger}$ Xingye Lu $\odot,{ }^{1, \star}$ and Pengcheng Dai $\odot^{3, \S}$

${ }^{1}$ Center for Advanced Quantum Studies and Department of Physics, Beijing Normal University, Beijing 100875, China

${ }^{2}$ School of Physical Science and Technology, ShanghaiTech University, Shanghai 201210, China

${ }^{3}$ Department of Physics and Astronomy, Rice University, Houston, Texas 77005, USA

${ }^{4}$ State Key Laboratory of Surface Physics, Department of Physics, Fudan University, Shanghai 200433, China

${ }^{5}$ Neutron Scattering Division, Oak Ridge National Laboratory, Oak Ridge, Tennessee 37831, USA

${ }^{6}$ ISIS Facility, Rutherford Appleton Laboratory, Chilton, Didcot, Oxfordshire OX11 OQX, United Kingdom

${ }^{7}$ Department of Physics, Royal Holloway University of London, Egham, TW20 OEX, United Kingdom

${ }^{8}$ School of Physics, Huazhong University of Science and Technology, Wuhan 430074, China

${ }^{9}$ Beijing Computational Science Research Center, Beijing 100094, China

${ }^{10}$ Department of Physics and HKU-UCAS Joint Institute for Theoretical and Computational Physics at Hong Kong, The University of Hong Kong, Hong Kong, China

(Received 23 December 2020; revised 30 March 2021; accepted 8 April 2021; published 27 May 2021)

Triangular lattice of rare-earth ions with interacting effective spin-1/2 local moments is an ideal platform to explore the physics of quantum spin liquids (QSLs) in the presence of strong spin-orbit coupling, crystal electric fields, and geometrical frustration. The $\mathrm{Yb}$ delafossites, $\mathrm{NaYbCh}_{2}(C h=\mathrm{O}, \mathrm{S}, \mathrm{Se})$ with $\mathrm{Yb}$ ions forming a perfect triangular lattice, have been suggested to be candidates for QSLs. Previous thermodynamics, nuclear magnetic resonance, and powder-sample neutron scattering measurements on $\mathrm{NaYbCh}_{2}$ have supported the suggestion of the QSL ground states. The key signature of a QSL, the spin excitation continuum, arising from the spin quantum number fractionalization, has not been observed. Here we perform both elastic and inelastic neutron scattering measurements as well as detailed thermodynamic measurements on high-quality single-crystal $\mathrm{NaYbSe}_{2}$ samples to confirm the absence of long-range magnetic order down to $40 \mathrm{mK}$, and further reveal a clear signature of magnetic excitation continuum extending from 0.1 to $2.5 \mathrm{meV}$. The comparison between the structure of the magnetic excitation spectra and the theoretical expectation from the spinon continuum suggests that the ground state of $\mathrm{NaYbSe}_{2}$ is a QSL with a spinon Fermi surface.

DOI: 10.1103/PhysRevX.11.021044

Subject Areas: Condensed Matter Physics, Magnetism, Strongly Correlated Materials

\section{INTRODUCTION}

The quantum spin liquid (QSL) is a correlated quantum state in a solid where the spins of the unpaired electrons are highly entangled over long distances, yet they do not

\footnotetext{
*gangchen.physics@gmail.com

†guoyf@shanghaitech.edu.cn

*luxy@bnu.edu.cn

§pdai@rice.edu

These authors contribute equally to this work.
}

Published by the American Physical Society under the terms of the Creative Commons Attribution 4.0 International license. Further distribution of this work must maintain attribution to the author(s) and the published article's title, journal citation, and DOI. exhibit any long-range magnetic order in the zero temperature limit. Originally proposed by Anderson as the ground state for a system of $S=1 / 2$ spins on a two-dimensional (2D) triangular lattice that interact antiferromagnetically with their nearest neighbors [1], a QSL is a novel quantum state of matter beyond the traditional Landau's symmetry breaking paradigm [2-5], and might be relevant for our understanding of high-temperature superconductivity [6-8] and quantum computation in certain cases $[9,10]$. Beyond the simple characterization of absence of a magnetic order, one key signature of the excitations in a QSL is the presence of deconfined spinons that are fractionalized quasiparticles carrying spin $1 / 2$, observed by inelastic neutron scattering as a spin excitation continuum 
fundamentally different from the integer spin-wave excitations in an ordered magnet [11-18].

Although spin excitation continuum has been observed in the geometrically frustrated $S=1 / 2$ single-crystal systems with 2D kagome [11], 2D triangular [12,13], three-dimensional distorted kagome bilayers [16], and 3D pyrochlore $[17,18]$ lattices, there is no consensus on the microscopic origin of the observed spin-excitation continuum. In the 2D $S=1 / 2$ kagome lattice $\mathrm{ZnCu}_{3}(\mathrm{OD})_{6} \mathrm{Cl}_{2}$ [11] and an effective $S=1 / 2$ triangular-lattice magnet $\mathrm{YbMgGaO}_{4}$ $[12-15,19,20]$, different interpretation of the observed spin-excitation continuum includes a spin glass state of magnetic [21] and nonmagnetic $\mathrm{Mg}-\mathrm{Ga}$ site disorder due to intrinsic sample issues [22-24], respectively, rather than the fractionalized quasiparticles of a QSL [5]. To conclusively identify the presence of deconfined spinon excitations in a QSL, one needs to search for the expected spin-excitation continuum among candidate QSL materials with highquality single crystals and establish their physical properties with clear experimental signatures and structures.

Recently, geometrically frustrated 2D triangular-lattice rare-earth-based materials with effective $S=1 / 2$ local moments have attracted considerable attention [25,26]. Compared with $\mathrm{YbMgGaO}_{4}$ [19], the family of $\mathrm{Yb}$
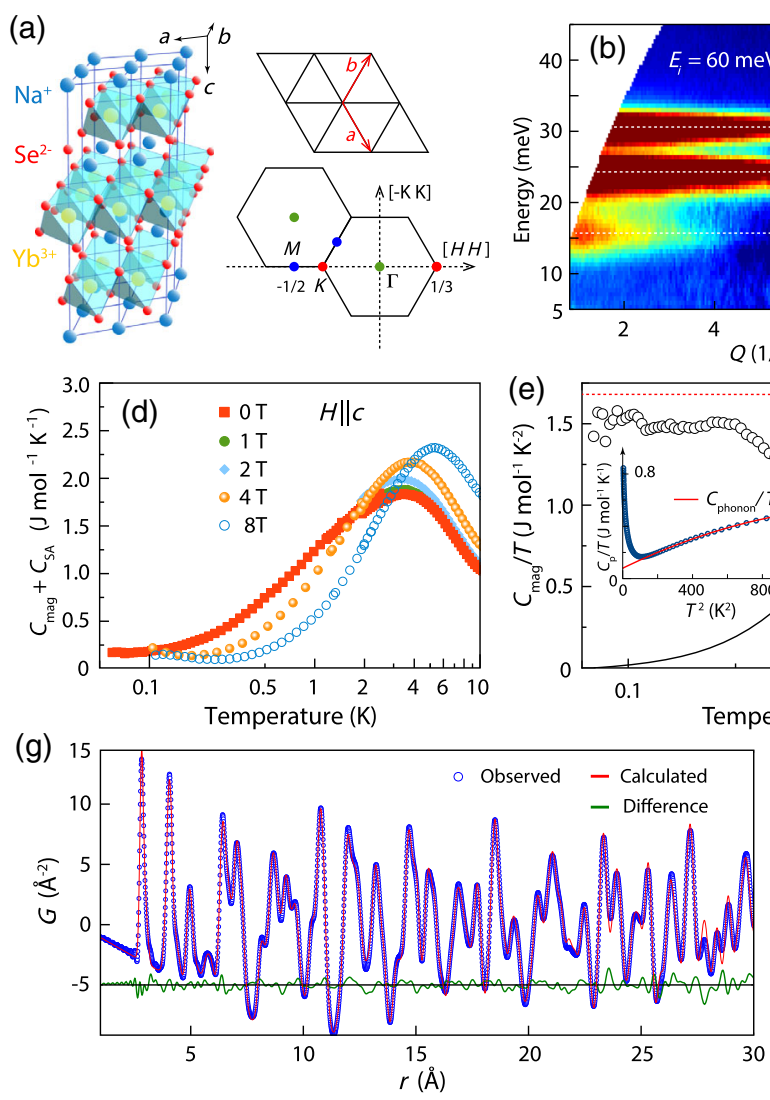
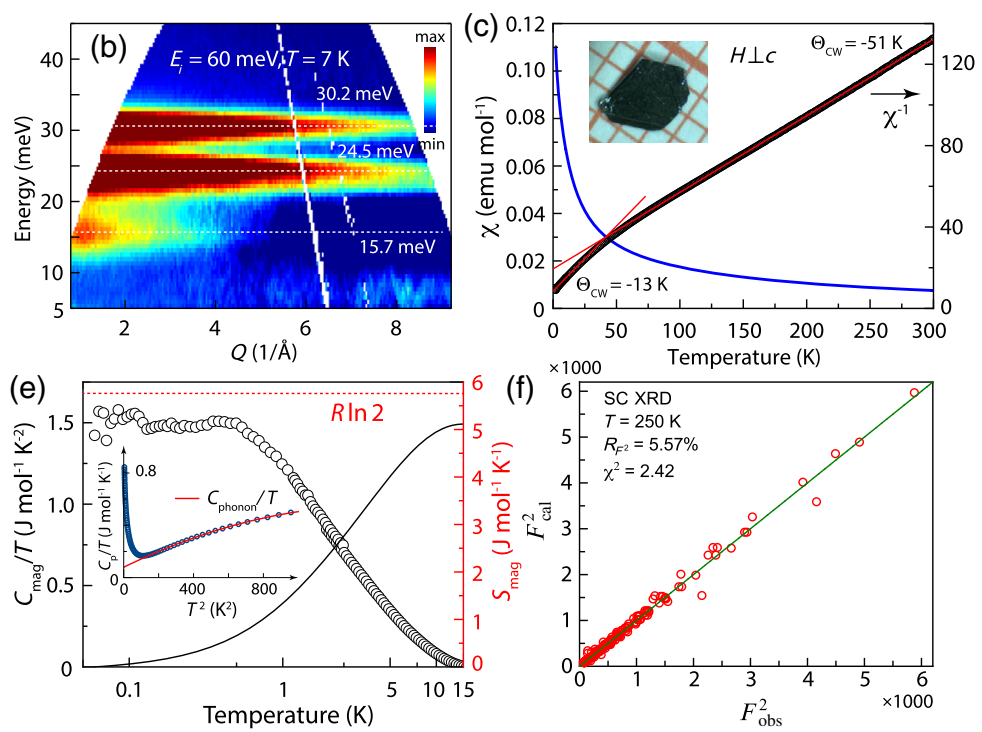

(f) $\times 1000$
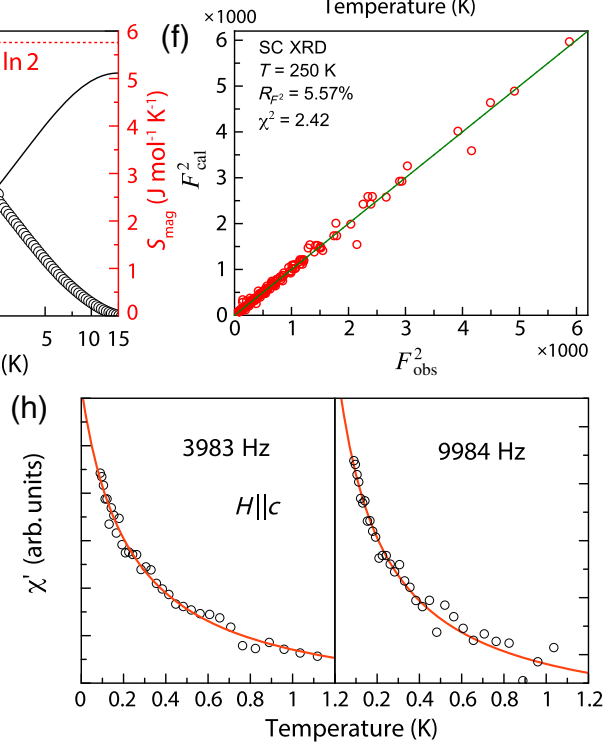

FIG. 1. Crystal structure and reciprocal space, CEF levels, heat capacity, and stoichiometry of $\mathrm{NaYbSe}_{2}$. (a) $\mathrm{The}_{\mathrm{Structure} \text { of } \mathrm{NaYbSe}}$ and corresponding reciprocal spac. The lattice parameters are $a=b \approx 4.07 \AA, c \approx 20.77 \AA$. (b) Inelastic neutron scattering spectra of $\mathrm{CEF}$ excitations obtained by subtracting the scattering of a nonmagnetic reference $\mathrm{NaYSe}_{2}$ from the intensity of $\mathrm{NaYbSe}_{2}$ (see the Appendix), in which the energy axis of $\mathrm{NaYSe}_{2}$ was shifted by $-1.7 \mathrm{meV}$ for calibrating the difference in phonon energies [38]. Three CEF energy levels are marked by white dashed lines. (c) Temperature-dependent magnetic susceptibility along $H \perp c$ direction. The fitting $\left(1 / \chi-\chi_{0}\right)=\left(C / T-\Theta_{\mathrm{CW}, \perp}\right)^{-1}$ for high-temperature range $(\sim 160-300 \mathrm{~K})$ results in a Curie-Weiss temperature $\Theta_{\mathrm{CW}, \perp} \approx$ $-51 \mathrm{~K}$, and the low-temperature range $(<20 \mathrm{~K})$ generates a $\Theta_{\mathrm{CW}, \perp} \approx-13 \mathrm{~K}$, in which $C$ is Curie constant, and $\chi_{0} \sim 2 \times 10^{-4} \mathrm{emu} / \mathrm{mol}$ is a temperature-independent background term. The inset shows the crystal for the magnetization measurements. (d) Temperaturedependent magnetic contribution $\left(C_{\mathrm{mag}}\right)$ to the specific heat (with minor contribution from nuclear $\mathrm{Schottky}$ anomaly $\left.C_{\mathrm{SA}}\right)$ of $\mathrm{NaYbSe}_{2}$ and its dependence on applied magnetic fields $H \| c$ [38]. Phonon contribution has been subtracted. (e) Temperature-dependent $C_{\text {mag }} / T$ (black circle) with $C_{\mathrm{SA}} / T$ subtracted [38] and the magnetic entropy (black curve). The red dashed line marks the value of $R$ ln 2 . The inset shows $C_{p} / T$ as a function of $T^{2}$. The red solid curve is a fitting of the phonon contribution $C_{\text {phonon. }}$ (f) The Rietveld refinement results of the single-crystal $\mathrm{x}$-ray diffraction (SC XRD) data at $250 \mathrm{~K}$ yield $\mathrm{Na}_{0.952(10)} \mathrm{Yb}_{0.048(10)} \mathrm{YbSe}_{2}$. $\mathrm{F}_{\text {cal }}^{2}$ and $\mathrm{F}_{\text {obs }}^{2}$ are the calculated and observed structure factors, respectively. (g) The PDF analysis of neutron data on $\mathrm{NaYbSe}_{2}$ up to $30 \AA$. The weighted residual value is $9.56 \%$. (h) ac susceptibility of $\mathrm{NaYbSe}_{2}$ single crystal measured with frequencies of 3983 and $9984 \mathrm{~Hz}$. The red solid curves are Curie-Weiss fits for the data. 
dichalcogenide delafossites $\mathrm{NaYbCh}_{2}(\mathrm{Ch}=\mathrm{O}, \mathrm{S}, \mathrm{Se})$ does not have the issue of $\mathrm{Mg}-\mathrm{Ga}$ site disorders in the nonmagnetic layers and thus provides a genuine example for an interacting spin-1/2 triangular-lattice antiferromagnet [27-29]. Moreover, $\mathrm{NaYbCh}_{2}$ exhibit larger magnetic anisotropy than $\mathrm{YbMgGaO}_{4}$ [12,30-33], suggesting that the in-plane magnetic interactions play the dominant role. The combination of the strong spin-orbit coupling and the crystal electric field (CEF) leads to a Kramers doublet ground state for the $\mathrm{Yb}^{3+}$ ion in $\mathrm{NaYbCh}_{2}$ that gives rise to the effective $S=1 / 2$ local moment at each ion site. Since the energy gaps between the ground and first excited Kramers doublets CEF levels for $\mathrm{NaYbSe}_{2}$ [Fig. 1(b)] [30], $\mathrm{NaYbS}_{2}$ [28], and $\mathrm{NaYbO}_{2}$ [29] are well above $\sim 12 \mathrm{meV}$, the magnetic properties below $100 \mathrm{~K}$ can be safely interpreted from the interaction between the effective $S=1 / 2$ local moments. Although previous experiments on powder samples of $\mathrm{NaYbO}_{2}$ provided some positive evidence for QSL ground states [34-37], there are no detailed neutron scattering experiments on single-crystal samples to establish the presence of the magnetic excitation continuum and further reveal its wave vector, energy, and temperature dependence, which are essential for identifying the nature of the possible QSL state. Here we report magnetic susceptibility, heat capacity, and neutron scattering results on single crystals of $\mathrm{NaYbSe}_{2}$. In addition to confirming the absence of magnetic order down to $40 \mathrm{mK}$ and spin freezing down to $90 \mathrm{mK}$, we show the presence of a spin-excitation continuum extending from 0.1 to $2.5 \mathrm{meV}$. Since our careful structure refinement and pair-distribution function (PDF) analysis reveal only $\sim 5 \%$ of $\mathrm{Yb}$ on $\mathrm{Na}$ site and no evidence for a spin glass state at $40 \mathrm{mK}$, we conclude that the ground state of $\mathrm{NaYbSe}_{2}$ has signatures of a QSL, consistent with the expectation of a spinon Fermi surface QSL state $[38,43]$.

\section{RESULTS}

High-quality single crystals of $\mathrm{NaYbSe}_{2}$ were grown by using flux method with Te as the flux (see the Appendix for further synthesis and experimental details). Figure 1(a) displays schematics of crystal structure and reciprocal space of $\mathrm{NaYbSe}_{2}$, where $\mathrm{Yb}$ ions form a perfect triangular lattice layer. Inelastic neutron scattering spectra of CEF excitations obtained by subtracting the scattering of a nonmagnetic reference $\mathrm{NaYSe}_{2}$ from $\mathrm{NaYbSe}_{2}$ are shown in Fig. 1(b) [38]. Consistent with previous work [30], the CEF levels of $\mathrm{Yb}^{3+}$ have a Kramers doublet ground state and three excited Kramers doublets at $E=15.7,24.5$, and $30.2 \mathrm{meV}$ at $T=7 \mathrm{~K}$, thus ensuring that all measurements below $\sim 100 \mathrm{~K}$ can be safely considered as an effective $S=$ $1 / 2$ ground state [30]. Note that the CEF levels with $E=$ 24.5 and $30.2 \mathrm{meV}$ are instrumental energy resolution limited [38] while the lowest CEF excitation is broader than the energy resolution indicative of the internal structure of this mode. The broadening could be attributed to the
$\mathrm{Yb}^{3+}-\mathrm{Yb}^{3+}$ exchange effects on CEF excitations that can split the lowest CEF excitation into two levels $[36,38]$.

To characterize the behavior of the local moments of $\mathrm{Yb}$ and their exchange interactions, we measured the magnetic susceptibility of single-crystal $\mathrm{NaYbSe}_{2}$. The temperature dependence of magnetization and the in-plane magnetic susceptibility $\chi_{\perp}(T)$ is depicted in Fig. 1(c), and a simple fit to the Curie-Weiss law yields $\Theta_{\mathrm{CW}, \perp} \simeq-13 \mathrm{~K}$ in the lowtemperature region $(<20 \mathrm{~K})$, whose absolute value is larger than $\left|\Theta_{\mathrm{CW}, \perp}\right| \simeq 7 \mathrm{~K}$ when the Van Vleck contribution is subtracted [44], indicating the predominantly antiferromagnetic spin interactions in $\mathrm{NaYbSe}_{2}$. Heat capacity measurements were also performed to characterize the thermodynamics of $\mathrm{NaYbSe}_{2}$, and the magnetic contribution $C_{\text {mag }}(T)$ to the specific heat of $\mathrm{NaYbSe}_{2}$ and its dependence on applied magnetic fields from 0 to $8 \mathrm{~T}$ are presented in Fig. 1(d). The data show a broad peak that shifts upward in temperature as a function of increasing magnetic field for $\mathrm{H} \| c$, no sharp anomaly indicative of the onset of long-range order, consistent with the susceptibility result and earlier work [44]. Figure 1(e) also shows the estimated temperature dependence of $C_{\text {mag }}(T) / T$ (left axis) and the corresponding magnetic entropy $S_{\text {mag }}$ (right axis). It is noted that $C_{\mathrm{mag}}(T) / T$ in the low-temperature regime $(<0.5 \mathrm{~K})$ is almost a constant, well compatible with the fact that the spinon Fermi surface alone has a constant density of states and would give a heat capacity depending linearly on temperature. Moreover, the temperature dependence of the magnetic entropy saturates to a value close to $S_{\mathrm{mag}} \approx$ $R \ln 2$ (where $R$ is the ideal gas constant) around $15 \mathrm{~K}$, consistent with an effective spin- $1 / 2$ description of the $\mathrm{Yb}^{3+}$ local moment [44].

Although stoichiometric $\mathrm{NaYbSe}_{2}$ has no intrinsic structural disorder in the $\mathrm{Na}^{+}$intercalating layer [27-29], real crystal could still have structural defects in $\mathrm{Na}^{+}$and $\mathrm{Se}^{2-}$ sites, and these vacant sites could be replaced by $\mathrm{Yb}^{3+}$ and $\mathrm{Te}^{2-}$, respectively (see the Appendix). To accurately determine the stoichiometry of our $\mathrm{NaYbSe}_{2}$, we carried out single-crystal $\mathrm{x}$-ray structural refinement by recording 1334 Bragg reflections, corresponding to 238 nonequivalent reflections. The Rietveld refinement results of the single-crystal X-ray diffraction data collected at $T=250 \mathrm{~K}$ are shown in Fig. 1(f), and the fitting outcome reveals full occupancy of the $\mathrm{Yb}^{3+}(3 a)$ and $\mathrm{Se}^{2-}(6 c)$ sites in the $\mathrm{YbSe}_{2}$ layers and $\sim 4.8 \% \pm 1 \%$ of the $\mathrm{Na}(3 b)$ sites occupied by the $\mathrm{Yb}$ ions. A small amount of $\mathrm{Yb}$ occupying the $\mathrm{Na}$ site is not surprising because $\mathrm{Yb}$ and $\mathrm{Na}$ have the same chemical environment for bonding, where both cations have six Se coordinates and are located at the center of $\mathrm{NaSe}_{6} / \mathrm{YbSe}_{6}$ octahedra [32]. To further characterize the structural character of the sample, we have also performed PDF analysis on neutron diffraction data measured on $2.7 \mathrm{~g}$ of $\mathrm{NaYbSe}_{2}$ powder ground from a large amount of single crystals obtained from the same batches as 
the spin-excitation measurements. As shown in Fig. 1(g), the local PDF peaks are well reproduced by fitting with the refined average structure using the x-ray diffraction data, indicating the absence of substantial local distortions. The average structure includes a $\mathrm{Yb}$ substitution at the $\mathrm{Na}$ site and possible excess Te at the Se site. The PDF analysis suggests an upper limit of $10 \%$ of $\mathrm{Yb}$ at the $\mathrm{Na}$ site and $0 \%$ $\mathrm{Te}$ at the Se site. While this value is larger than that obtained by single-crystal x-ray refinements, single-crystal refinement results are more accurate as more Bragg peaks are measured in the x-ray refinements. These results are consistent with the inductively coupled plasma measurements of chemical composition of the sample (see the Appendix for details). Although $\mathrm{Yb}$ ions in the $\mathrm{Na}$ layers may be magnetic, our frequency-dependent ac susceptibility measurements down to $90 \mathrm{mK}$ can be well described with a Curie-Weiss fit and show no evidence of spin freezing [Fig. 1(h)].

In the previous inelastic neutron scattering measurements on single crystals of $\mathrm{CsYbSe}_{2}\left(\Theta_{\mathrm{CW}} \simeq-13 \mathrm{~K}\right)$, spin excitations were found to be centered around the $K$ point in reciprocal space [Fig. 1(a)], with no intensity modulation along the $c$ axis, and extending up to $1 \mathrm{meV}$ [45]. To determine what happens in $\mathrm{NaYbSe}_{2}$, we must first determine if the system has long- or short-range magnetic order. For this purpose, we align the crystals in the $[H, H, 0] \times[0,0, L]$ and $[H, 0,0] \times[0, K, 0]$ zones [Fig. 1(a)]. Figures 2(a) and 2(b) display maps of elastic scattering in the $[H, H, L]$ and $[H, K, 0]$ planes, respectively, at $T=40 \mathrm{mK}$ (top panels) and $40 \mathrm{mK}-10 \mathrm{~K}$ (bottom panels). In both cases, no evidence of long- or short-range magnetic order was observed at $40 \mathrm{mK}$, consistent with previous magnetic susceptibility, heat capacity, and nuclear magnetic resonance measurements [44]. The wave vector dependence of the spin excitations of $E=$ $0.3 \pm 0.1 \mathrm{meV}$ in the $[H, H, L]$ zone at $40 \mathrm{mK}$ (left-hand panel) and $10 \mathrm{~K}$ (right-hand panel) is presented in Fig. 2(c). At $40 \mathrm{mK}$, one can see a featureless rod of scattering along the $[1 / 3,1 / 3, L]$ direction, indicating that spin excitations in $\mathrm{NaYbSe}_{2}$ are 2D in nature and have no $c$-axis modulations. The scattering becomes much weaker at $10 \mathrm{~K}$, thus confirming the magnetic nature of the scattering at $40 \mathrm{mK}$. Moreover, Fig. 2(d) shows the temperature dependence of the $E=0.3 \pm 0.1 \mathrm{meV}$ spin excitations in the $[H, K, 0]$ zone. The magnetic scattering is centered around the $K$ point, consistent with the previous work [45], and decreases significantly with increasing temperature.

To further reveal the intrinsic quantum dynamics of the local moments of the $\mathrm{Yb}$ ions, we perform the inelastic neutron scattering measurements to study the spin excitations in single crystals of $\mathrm{NaYbSe}_{2}$ at both $40 \mathrm{mK}$ and $10 \mathrm{~K}$. Constant-energy images of spin excitations with a variety of energies in the in-plane $2 \mathrm{D}$ Brillouin zones at $40 \mathrm{mK}$ and $10 \mathrm{~K}$ are summarized in Figs. 3(a)-3(d) and Figs. 3(e)-3(h), respectively. At $E=0.15 \pm 0.05 \mathrm{meV}$ and
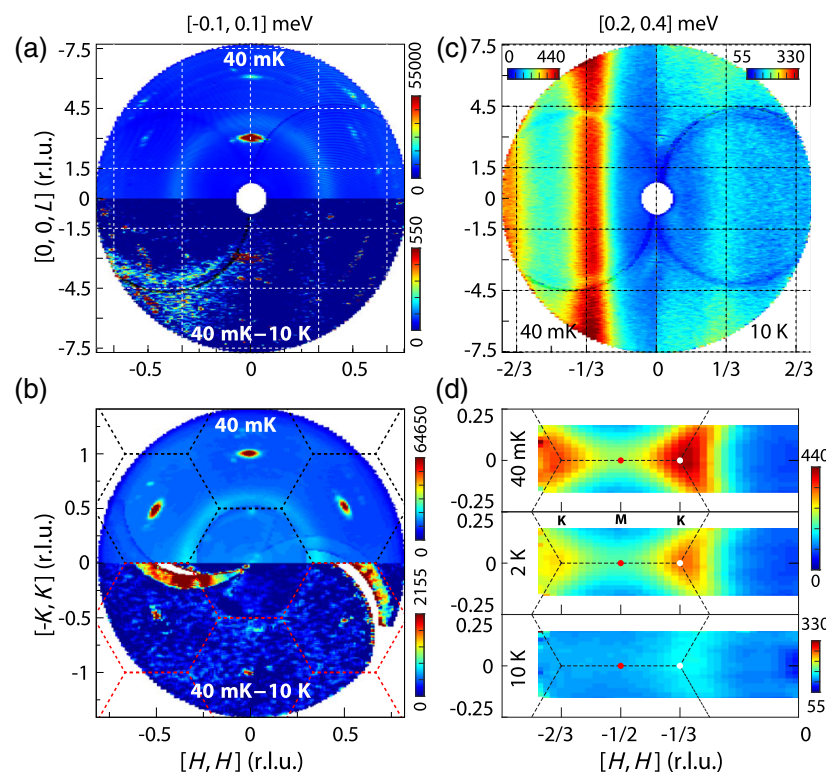

(d)

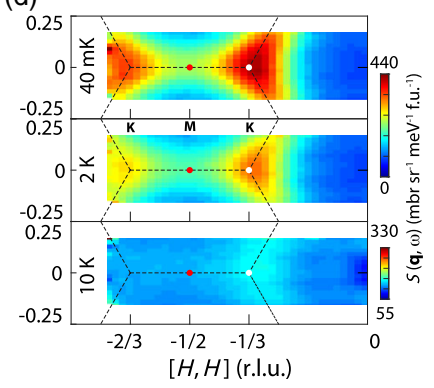

FIG. 2. Neutron scattering results in $[H, H, L]$ and $[H, K, 0]$ zones. Elastic neutron scattering results $(E=0 \pm 0.1 \mathrm{meV})$ in (a) the $[H, H, L]$ plane and (b) $[H, K, 0]$ plane measured with $E_{i}=$ 3.32 and $3.70 \mathrm{meV}$, respectively. Scattering along the vertical direction $[[-K, K, 0]$ for (a) and $[0,0, L]$ for (b) $]$ is integrated. The upper half panels of (a) and (b) are data at $T=40 \mathrm{mK}$, and the lower are the differences between $T=40 \mathrm{mK}$ and $10 \mathrm{~K}$. (c) $L$ dependence of the spin excitations along the $[H, H]$ direction at $T=40 \mathrm{mK}$ (left half panel) and $T=10 \mathrm{~K}$ (right half panel), with $K=[-0.05,0.05]$ and $E=0.3 \pm 0.1 \mathrm{meV}$. (d) Spin excitations with $E=0.3 \pm 0.1$ in the $[H, K]$ plane measured at $T=40 \mathrm{mK}, 2$, and $10 \mathrm{~K}$. Scattering along the $[0,0, L]$ direction is integrated. The black dashed lines mark the Brillouin zones of $\mathrm{NaYbSe}_{2}$.

$40 \mathrm{mK}$, the magnetic scattering spectral weights spread broadly in the Brillouin zone but with higher intensity at the $K$ point and no scattering near the zone center (the $\Gamma$ point) [Fig. 3(a)]. This is clearly different from the wave vector dependence of the low-energy magnetic scattering for $\mathrm{YbMgGaO}_{4}$, in which the spectral weight is enhanced around the $M$ point [12]. The high intensity at the $K$ point in $\mathrm{NaYbSe}_{2}$ might arise from the strong $X Y$-type exchange interaction, since the strong spin-orbit coupling in this material indeed brings certain anisotropic interactions [46]. With increasing energies to $E=0.6 \pm 0.1$ [Fig. 3(b)], $1.1 \pm 1$ [Fig. 3(c)], and $E=2.1 \pm 0.1 \mathrm{meV}$ [Fig. 3(d)], the magnetic scattering spectral weights become more evenly distributed in the Brillouin zone and gradually decrease with increasing energy. While the spin-excitation continuum at $E=0.15 \pm 0.05 \mathrm{meV}$ nearly vanishes on warming from $40 \mathrm{mK}$ to $10 \mathrm{~K}$ [Fig. 3(e)], the spectral weights at other energies become weaker but are still located around the Brillouin zone boundaries, especially the scattering at the $K$ points [Figs. 3(f) and $3(\mathrm{~g})$ ].

Figures 4(c) and 4(d) display the wave vector energy dependence of the spin excitation spectral intensity (in $\log$ scale) along the magenta color arrow direction in 

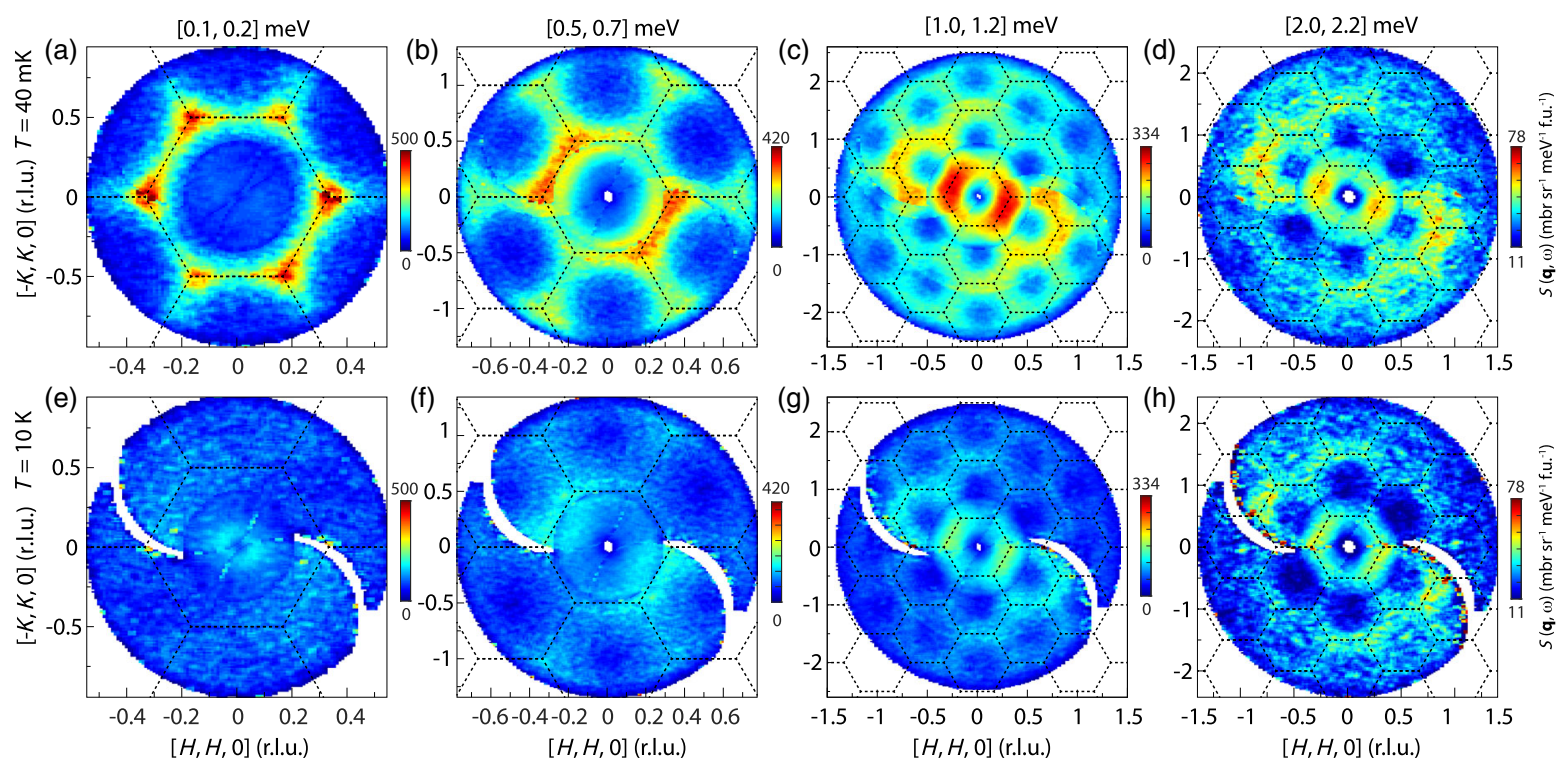

FIG. 3. Constant-energy images of spin excitations in the $[H, K, 0]$ plane. (a)-(d) Images at $T=40 \mathrm{mK}$ and (e)-(h) $10 \mathrm{~K}$. The intensity along the vertical $[0,0, L]$ direction is integrated. Spin excitations for (a),(e) $E=0.15 \pm 0.05$, (b),(f) $0.6 \pm 0.1,(\mathrm{c}),(\mathrm{g})$ $1.1 \pm 0.1$, and (d),(h) $2.1 \pm 0.1 \mathrm{meV}$ are measured with $E_{i}=1.77,3.70,12.14$, and $12.14 \mathrm{meV}$, respectively. The black dashed lines mark the Brillouin zones in the reciprocal space. The data are collected in $180^{\circ}$ range of sample rotation around the $c$ axis. The $360^{\circ}$ circular coverage is generated by averaging the raw data and its mirror in the $[H, K, 0]$ plane. The $C_{2}$-like anisotropy has been attributed to a trivial effect caused by sample-volume change in beam during sample rotation for neutron scattering measurements in $[H, K, 0]$ plane [38].
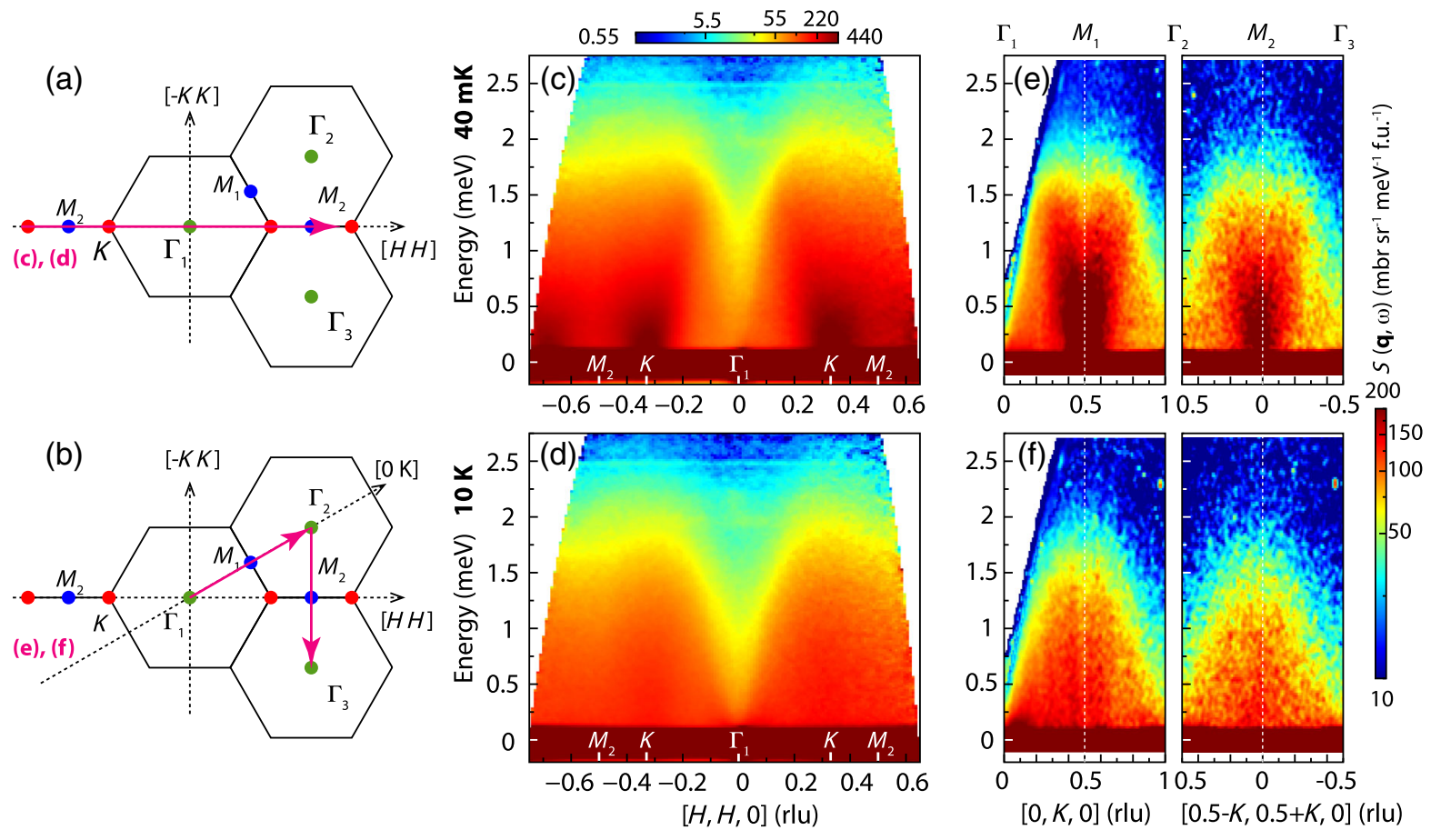

FIG. 4. Spin-excitation spectra along high symmetry momentum directions. (a),(b) Schematics of the Brillouin zones with high symmetry points $\Gamma, K$, and $M$ denoted by green, red, and blue dots, respectively, and the high symmetry directions for the images in (c)-(f) marked by pink lines with arrow heads. Spin-excitation spectra collected at (c) $T=40 \mathrm{mK}$ and (d) $10 \mathrm{~K}$ along the $M_{2}-K-\Gamma-K-M_{2}$ with $E_{i}=3.32 \mathrm{meV}$. (e),(f) Intensity color maps along the $\Gamma_{1}-M_{1}-\Gamma_{2}$ and $\Gamma_{2}-M_{2}-\Gamma_{3}$ directions measured with $E_{i}=3.7 \mathrm{meV}$. 
Fig. 4(a) at $40 \mathrm{mK}$ and $10 \mathrm{~K}$, respectively. The excitation continuum here is analogous to that calculated from the free spinon theory. The excitation bandwidth $(\sim 2 \mathrm{meV})$, together with the Curie-Weiss temperatures, characterize the scale of the magnetic interactions [12,43]. At both $40 \mathrm{mK}$ and $10 \mathrm{~K}$, the spectral intensity is broadly distributed in the energy-momentum plane, and the excitation intensity gradually decreases with increasing energy and finally vanishes above $\sim 2.2 \mathrm{meV}$. The broad neutron scattering spectral intensity at $40 \mathrm{mK}$ persists to the lowest energy that we measured, implying a high density of spinon scattering states at low energies. Moreover, the spectral weight around the $\Gamma$ point is suppressed to form a $\mathrm{V}$-shaped upper bound [38]. Combining these two facts, it strongly suggests a spinon Fermi surface QSL since this scenario not only provides a high density of spinon states near the Fermi surface, but also well explains the V-shaped upper bound on the excitation energy near the $\Gamma$ point [43].

The $\mathrm{V}$-shaped structure is one of the key properties for the magnetic excitation in the spinon Fermi surface QSL. It arises from the large density of states and the linear $E-k$ spinon dispersion near the Fermi surface. Because of the
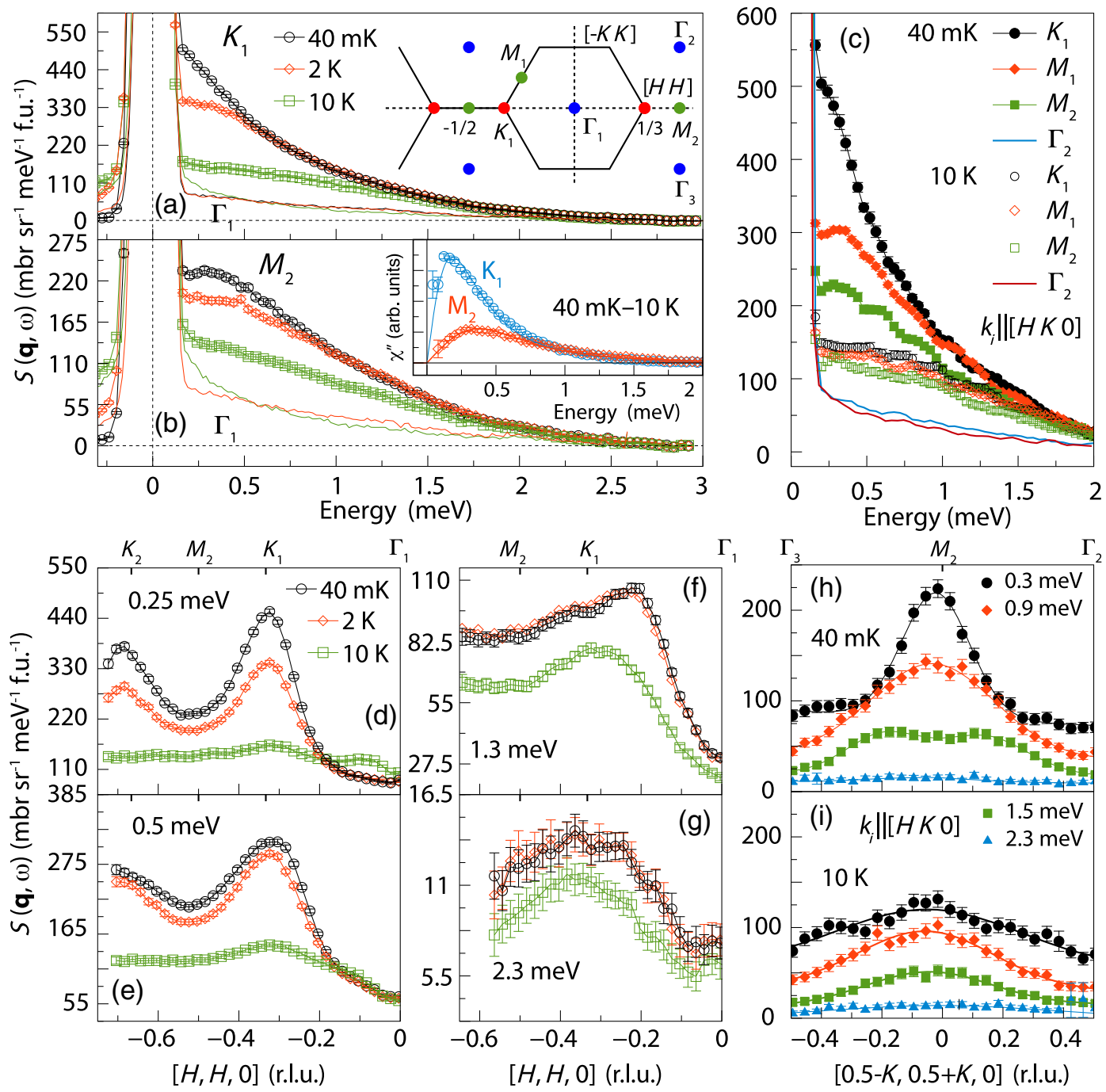

FIG. 5. Wave vector dependence of spin excitations along high symmetry directions. The wave vector cuts in (a),(b),(d)-(g) were measured in the $[H, H, L]$ zone with $E_{i}=3.32 \mathrm{meV}$, while those in (c),(h),(i) were measured in the $[H, K, 0]$ plane with $E_{i}=3.70 \mathrm{meV}$. Panels (a) and (b) show the energy-dependent scattering at $K_{1}$ and $M_{1}$ points measured at $T=40 \mathrm{mK}$ (black circle), $2 \mathrm{~K}$ (red diamond), and $10 \mathrm{~K}$ (green square). The inset in (a) is a schematic of the reciprocal space with the $\Gamma, K$, and $M$ points denoted by green, red, and blue dots, respectively. The black, red, and green curves are energy cuts at $\Gamma_{1}$. The inset of (b) shows the difference of $\chi^{\prime \prime}$ between the spectra for $T=40 \mathrm{mK}$ and $10 \mathrm{~K}$ at the $K$ and $M$ points. The light blue and red curves are fittings of the $\chi^{\prime \prime}$ with a damped harmonic oscillator model. Panel (c) shows the energy cuts at the $K_{1}, M_{1}, M_{2}$, and $\Gamma_{2}$. Solid symbols represent the data collected at $T=40 \mathrm{mK}$ and the open symbols collected at $10 \mathrm{~K}$. The black and blue curves are energy cuts at the $\Gamma_{2}$ point measured at $T=40 \mathrm{mK}$ and $10 \mathrm{~K}$, respectively. (d)-(g) Constant energy cuts along the $M_{2}-K_{1}-\Gamma_{1}$ for $T=40 \mathrm{mK}, 2 \mathrm{~K}$, and $10 \mathrm{~K}$, with corresponding energy transfers marked in the panels. Constant energy cuts along the $\Gamma_{3}-M_{2}-\Gamma_{2}$ measured at (h) $T=40 \mathrm{mK}$ and (i) $10 \mathrm{~K}$. The solid curves are guides to the eyes, and the error bars represent one standard deviation. 
spin quantum number fractionalization, the neutron scattering creates the spinon particle-hole pairs across the spinon Fermi surface. To excite the pair with an energy $E$, a minimal momentum transfer $E / v_{F}$ is needed where $v_{F}$ is the Fermi velocity. The slope of the $\mathrm{V}$ shape is expected to be the Fermi velocity. It is also noted that the low-energy spin excitations clearly peak around the $K$ point at $40 \mathrm{mK}$ [Fig. 4(c)], and they decrease dramatically on warming but still keep the $\mathrm{V}$-shaped upper bound around the $\Gamma$ point at $10 \mathrm{~K}$ [Fig. 4(d)]. In addition, Figs. 4(e) and 4(f) present the wave vector energy dependence of the spin-excitation spectral intensity along the magenta color arrow directions in Fig. 4(b) at $40 \mathrm{mK}$ and $10 \mathrm{~K}$, respectively. The main results are similar to that in Figs. 4(c) and 4(d), and also support a spinon Fermi surface QSL.

The data points in Figs. 5(a) and 5(b) show energy dependence of spin excitations at the $K_{1}$ and $M_{2}$ points, respectively, under a variety of temperatures $T=40 \mathrm{mK}$, $2 \mathrm{~K}$, and $10 \mathrm{~K}$. The solid lines in the figures display similar data at the $\Gamma_{1}$ point. Consistent with Fig. 4, magnetic scattering clearly decreases with increasing temperature at the $K_{1}$ and $M_{2}$ points, and essentially vanishes at the $\Gamma_{1}$ point. The temperature differences $(40 \mathrm{mK}-10 \mathrm{~K})$ of the imaginary part of the dynamic susceptibility, $\chi^{\prime \prime}(E)$, at the $K_{1}$ and $M_{2}$ points peak around 0.15 and $0.3 \mathrm{meV}$, respectively, as shown in the inset in Fig. 5(b). Additionally, Fig. 5(c) compares energy dependence of the magnetic scattering at the $M_{1}, M_{2}$, and $K_{1}$ with the background at the $\Gamma_{2}$ point. To show the wave vector dependence of spin excitations, Figs. 5(d)-5(g) plot the spectral intensity along the $[H, H, 0]$ direction for various energies of $E=$ $0.25 \pm 0.1, \quad 0.5 \pm 0.1, \quad 1.3 \pm 0.1, \quad$ and $2.3 \pm 0.1 \mathrm{meV}$, respectively, at $T=40 \mathrm{mK}, 2 \mathrm{~K}$, and $10 \mathrm{~K}$. Similarly, Figs. 5(h) and 5(i) also plot constant-energy cuts along the $[0.5-K, 0.5+K, 0]$ direction for energies of $E=0.3 \pm 0.1, \quad 0.9 \pm 0.1, \quad 1.5 \pm 0.1, \quad 2.3 \pm 0.1 \mathrm{meV}$ at $40 \mathrm{mK}$ and $10 \mathrm{~K}$, respectively. All the results are compatible with Figs. 4(c)-4(f). In Figs. 5(a) and 5(b), the spin excitations can only be resolved above $E \sim 0.15 \mathrm{meV}$ because of the instrumental energy resolution. To further

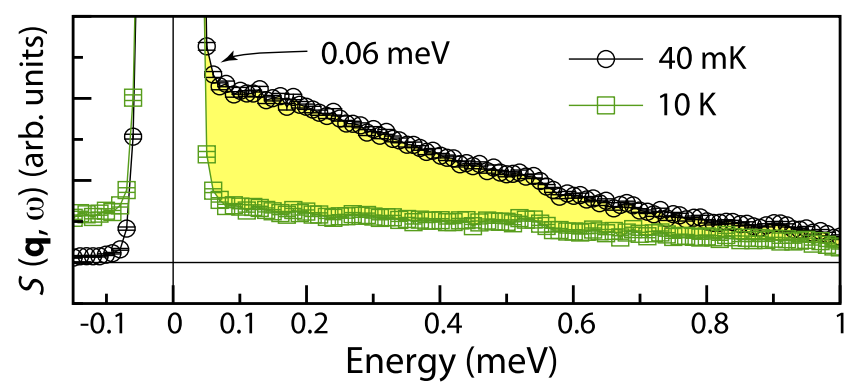

FIG. 6. Spin-excitation energy spectra at $K_{1}$ position measured with $E_{i}=1.55 \mathrm{meV}$ at $T=40 \mathrm{mK}$ (black circles) and $10 \mathrm{~K}$ (green squares). The yellow shaded area marks the difference between the spectra for $T=40 \mathrm{mK}$ and $10 \mathrm{~K}$. The black arrow marks the lowest energy $(0.06 \mathrm{meV})$ magnetic excitations. check whether the excitations are gapless, we show in Fig. 6 spin-excitation energy spectra at the $K_{1}$ point measured with improved instrumental energy resolution $\left(E_{i}=1.55 \mathrm{meV}\right)$. The energy-dependent spin excitations for $T=40 \mathrm{mK}$ and $10 \mathrm{~K}$ reveal the persistence of the spin excitations down to $E \sim 0.06 \mathrm{meV}$, indicative of the gapless nature for the excitations [38].

\section{DISCUSSION AND CONCLUSION}

Overall, the magnetic and heat capacity measurements, combined with the neutron scattering results on single crystals of $\mathrm{NaYbSe}_{2}$, demonstrate the absence of long- or short-range magnetic order even down to $40 \mathrm{mK}$, implying a quantum disordered QSL state. In particular, besides the naive disorder and the simple spectral continuum of spin excitation, the almost linear temperature dependence of magnetic heat capacity $C_{\mathrm{mag}}(T)$ at the low-temperature regime, the enormous low-energy gapless excitations, and the $\mathrm{V}$-shaped upper bound around the $\Gamma$ point in inelastic neutron scattering spectrum all strongly indicate the existence of a spinon Fermi surface. Theoretically, although the pure compact $\mathrm{U}(1)$ gauge theory in two spatial dimensions is always confined due to the nonperturbative instanton events [47], it has been shown and understood that in the presence of spinon Fermi surface and gapless excitations, the QSL phase could be stable against gauge fluctuations, and a noncompact $\mathrm{U}(1)$ gauge theory remains to be a good low-energy description $[8,48]$. Therefore, our experimental results and conclusion about spinon Fermi surface QSL can be compatible with theory. The scenario of spinon Fermi surface QSL could further be verified by low-temperature thermal-transport measurement, which has an advantage to unveil the nature of low-energy itinerant excitations.

Very recently, the pressure-induced insulator to metal transition followed by an emergence of superconductivity in $\mathrm{NaYbSe}_{2}$ was observed in experiments [49,50]. This is quite remarkable since the QSL has long been thought to be a parent state of the high-temperature superconductivity [6-8]. It was suggested that doping a QSL could naturally result in superconductivity [6-8] due to the intimate relationship between high-temperature superconductivity and QSL, but the definitive experimental evidence showing that doping QSLs give rise to superconductivity is still lacking. Instead of doping, Refs. [49,50] obtained the superconductivity by pressure, which opens up a promising way to study the superconductivity in QSL candidates and sheds light on the mechanism of high-temperature superconductivity.

\section{ACKNOWLEDGMENTS}

We thank M. Stone for suggestions of appropriate neutron scattering instrumentation, and Feng Ye (ORNL) for the assistance with the single-crystal $\mathrm{x}$-ray diffraction measurements. We also thank Ling Wang and Yuesheng $\mathrm{Li}$ for helpful discussions. The research at Beijing Normal 
University is supported by the National Natural Science Foundation of China (Grants No. 11734002 and No. 11922402). The work at ShanghaiTech university is supported by the National Natural Science Foundation of China (No. 11874264, Y. Guo). Y. Guo and X. W. thank the support from Analytical Instrumentation Center (No. SPSTAIC10112914), SPST, ShanghaiTech University. The neutron scattering work at Rice is supported by U.S. DOE BES DE-SC0012311 (P. D.). This work is further supported by funds from the Ministry of Science and Technology of China (Grants No. 2016YFA0301001, No. 2018YFGH000095, No. 2016YFA0300500 for G.C., and Grants No. 2016YFA0300501 and No. 2016YFA0300503 for L. S. and G. C.) and from the Research Grants Council of Hong Kong with General Research Fund Grant No. 17303819 (G. C.). E. F. and H. C. acknowledges support of U.S. DOE BES Early Career Award No. KC0402010 under Contract No. DE-AC05-00OR22725. E. M. and C.L. H. acknowledge support from U.S. DOE BES DESC0019503. This research used resources at Spallation Neutron Source, a U.S. DOE Office of Science User Facility operated by ORNL. We gratefully acknowledge the Science and Technology Facilities Council (STFC) for access to neutron beam time at ISIS.

\section{APPENDIX: METHODS}

Crystal growth.-All the $\mathrm{NaYbSe}_{2}$ single crystals used in this study were grown by using Te as the flux. The starting materials are in a molar ration of $\mathrm{Na}: \mathrm{Yb}: \mathrm{Se}: \mathrm{Te}=1: 1: 2: 20$. To avoid the violent reaction between $\mathrm{Na}$ and $\mathrm{Se}$, the $\mathrm{Na}(99.7 \%)$ blocks and $\mathrm{Te}$ $(99.999 \%)$ granules were mixed and slowly heated up to $200^{\circ} \mathrm{C}$ within $20 \mathrm{~h}$ and prereacted at the temperature for $10 \mathrm{~h}$. The precursor was then thoroughly mixed with $\mathrm{Yb}$ (99.9\%) blocks and Se (99.999\%) granules in the molar ratio and placed into an alumina crucible. The crucible was sealed into a quartz tube under the vacuum of $10^{-4} \mathrm{~Pa}$ and then slowly heated up to $950^{\circ} \mathrm{C}$ within $15 \mathrm{~h}$. After the reaction at this temperature for $20 \mathrm{~h}$, the assembly was slowly cooled down to $800^{\circ} \mathrm{C}$ at a temperature decreasing rate of $1{ }^{\circ} \mathrm{C} / \mathrm{h}$. At $800{ }^{\circ} \mathrm{C}$, the quartz tube was immediately taken out of the furnace and placed into a high-speed centrifuge to separate the excess Te flux. To show a comparison, $\mathrm{NaYbSe}_{2}$ crystals were also grown by using $\mathrm{NaCl}$ as the flux in a similar procedure as mentioned above (not used for this study) [38]. The crystallographic phase and quality of the grown crystals were examined on a Bruker D8 VENTURE single-crystal x-ray diffractometer using Mo $K_{\alpha 1}$ radiation $(\lambda=0.71073 \AA)$ at room temperature. The crystals grown by using different flux have the same high quality [38]. Growth of the polycrystalline $\mathrm{NaYbSe}_{2}$ and $\mathrm{NaYSe}_{2}$ samples has been described elsewhere [44].

Stoichiometric analysis. - The single-crystal x-ray diffraction of $\mathrm{NaYbSe}_{2}$ was performed at $250 \mathrm{~K}$ on the Rigaku
XtaLAB PRO diffractometer at Spallation Neutron Source, Oak Ridge National Laboratory (ORNL). Structure refinement based on the x-ray diffraction data were carried out with the FullProf suite [51], generating $\left(\mathrm{Na}_{0.952(10)} \mathrm{Yb}_{0.048(10)}\right) \mathrm{YbSe}_{2}$ without $\mathrm{Te}$ occupying $\mathrm{Se}$ sites. Elemental analysis of a group of $\mathrm{NaYbSe}_{2}$ single crystals grown with Te flux with a total mass of $35 \mathrm{mg}$ was performed by the inductively coupled plasma (ICP) method on the Thermo Fisher ICP 7400 system. The result $-\mathrm{Na}_{0.965} \mathrm{Yb}_{1.03} \mathrm{Se}_{1.98} \mathrm{Te}_{0.025}$ - can be interpreted as $\sim 3 \%$ of $\mathrm{Na}^{+}$sites being occupied by $\mathrm{Yb}$ ions and agrees well with the structure refinement results of single-crystal $\mathrm{x}$-ray diffraction, especially considering that Te could exist as flux in the sample.

Heat capacity.-The specific heat capacity of $\mathrm{NaYbSe}_{2}$ was measured down to $50 \mathrm{mK}$ using a thermal-relaxation method in DynaCool-PPMS (Physical Property Measurement System, Quantum Design) with the magnetic field applied along the $c$ axis at Fudan University and Rice University. The total specific heat is described as a sum of magnetic and lattice contributions: $C_{p}=C_{\mathrm{mag}}+C_{\text {phonon }}$. We fit the phonon contribution with $C_{\text {phonon }}=\beta T^{3}+\alpha T^{5}$.

Magnetic susceptibility.-The magnetic susceptibility of a rare-earth magnetic system with strong spin-orbit coupling can be determined by CEF excitations, particularly the first CEF excitation level. In this case, the Curie-Weiss analysis is applicable only in the limited temperature range much smaller than the first CEF excitation level. In $\mathrm{NaYbSe}_{2}$, spin-orbit coupling is quite a large energy scale and generates the local moment $J=7 / 2$ for $\mathrm{Yb}^{3+}$ ion. The crystal field further splits the eight $J=7 / 2$ states into 4 Kramers doublets, and the ground state doublet contributes to the effective spin- $1 / 2$ description that is responsible for the low-temperature magnetism. Since the lowest CEF excitation is $\sim 15.7 \mathrm{meV}(\sim 180 \mathrm{~K})$, the Curie-Weiss fitting of the $T<20 \mathrm{~K}$ range is not affected by spin-orbit coupling and CEF levels.

Neutron scattering.-The neutron scattering measurements of the magnetic excitations in the $[H, H, L]$ scattering plane and the CEF excitations were performed on the ColdNeutron-Chopper-Sepctrometer (CNCS) [52] and ARCS [53] at the Spallation Neutron Source, ORNL, respectively. The measurements in the $[H, K, 0]$ scattering plane were carried out on the LET cold neutron chopper spectrometer [54,55], ISIS spallation neutron source, Rutherford Appleton Laboratory (RAL), UK. We coaligned $\sim 3.7 \mathrm{~g}$ of $\mathrm{NaYbSe}_{2}$ single crystals for the measurements of magnetic excitations and prepared $\sim 10 \mathrm{~g} \mathrm{NaYbSe}_{2}$ and $\mathrm{NaYSe}_{2}$ polycrystalline samples for the CEF excitation measurements. The powder neutron diffraction experiment for pair-distribution function analysis was performed at NOMAD, ORNL at $100 \mathrm{~K}$, with $2.7 \mathrm{~g}$ of $\mathrm{NaYbSe}_{2}$ polycrystalline sample ground from $\sim 100$ pieces of single crystals obtained from the same batches as the $3.7 \mathrm{~g}$ sample set for our elastic or inelastic neutron 
scattering experiments at CNCS and LET. The neutron scattering data were reduced with Mantid [56] and analyzed with MantidPlot, HORACE [57], and MSlice.

[1] P. W. Anderson, Resonating Valence Bonds: A New Kind of Insulator?, Mater. Res. Bull. 8, 153 (1973).

[2] L. Balents, Spin Liquids in Frustrated Magnets, Nature (London) 464, 199 (2010).

[3] Y. Zhou, K. Kanoda, and T.-K. Ng, Quantum Spin Liquid States, Rev. Mod. Phys. 89, 025003 (2017).

[4] L. Savary and L. Balents, Quantum Spin Liquids: A Review, Rep. Prog. Phys. 80, 016502 (2017).

[5] C. Broholm, R J. Cava, S. A. Kivelson, D. G. Nocera, M. R. Norman, and T. Senthil, Quantum Spin Liquids, Science 367, eaay0668 (2020).

[6] P. W. Anderson, The Resonating Valence Bond State in $\mathrm{La}_{2} \mathrm{CuO}_{4}$ and Superconductivity, Science 235, 1196 (1987).

[7] P. A. Lee, N. Nagaosa, and X. G. Wen, Doping a Mott Insulator: Physics of High-Temperature Superconductivity, Rev. Mod. Phys. 78, 17 (2006).

[8] P. A. Lee, From High Temperature Superconductivity to Quantum Spin Liquid: Progress in Strong Correlation Physics, Rep. Prog. Phys. 71, 012501 (2008).

[9] A. Y. Kitaev, Fault-Tolerant Quantum Computation by Anyons, Ann. Phys. (Amsterdam) 303, 2 (2003).

[10] A. Y. Kitaev, Anyons in an Exactly Solved Model and Beyond, Ann. Phys. (Amsterdam) 321, 2 (2006).

[11] T. H. Han, J. S. Helton, S. Chu, D. G. Nocera, J. A. Rodriguez-Rivera, C. Broholm, and Y. S. Lee, Fractionalized Excitations in the Spin-Liquid State of a KagomeLattice Antiferromagnet, Nature (London) 492, 406 (2012).

[12] Y. Shen et al., Evidence for a Spinon Fermi Surface in a Triangular-Lattice Quantum-Spin-Liquid Candidate, Nature (London) 540, 559 (2016).

[13] J. A. M. Paddison, M. Daum, Z. Dun, G. Ehlers, Y. Liu, M. B. Stone, H. Zhou, and M. Mourigal, Continuous Excitations of the Triangular-Lattice Quantum Spin Liquid $\mathrm{YbMgGaO}_{4}$, Nat. Phys. 13, 117 (2017).

[14] Y. Li, D. Adroja, D. Voneshen, R. I. Bewley, Q. Zhang, A. A. Tsirlin, and P. Gegenwart, Nearest-Neighbour Resonating Valence Bonds in $\mathrm{YbMgGaO}_{4}$, Nat. Commun. 8, 15814 (2017).

[15] Y. Li, S. Bachus, B. Liu, I. Radelytskyi, A. Bertin, A. Schneidewind, Y. Tokiwa, A. A. Tsirlin, and P. Gegenwart, Rearrangement of Uncorrelated Valence Bonds Evidenced by Low-Energy Spin Excitations in $\mathrm{YbMgGaO}_{4}$, Phys. Rev. Lett. 122, 137201 (2019).

[16] C. Balz et al., Physical Realization of a Quantum Spin Liquid Based on a Complex Frustration Mechanism, Nat. Phys. 12, 942 (2016).

[17] B. Gao et al., Experimental Signatures of a Three-Dimensional Quantum Spin Liquid in Effective Spin-1/2 $\mathrm{Ce}_{2} \mathrm{Zr}_{2} \mathrm{O}_{7}$ Pyrochlore, Nat. Phys. 15, 1052 (2019).

[18] J. Gaudet, E. M. Smith, J. Dudemaine, J. Beare, C. R. C. Buhariwalla, N. P. Butch, M. B. Stone, A. T. Kolesnikov, G. $\mathrm{Xu}, \mathrm{D} . \mathrm{R}$. Yahne et al., Quantum Spin Ice Dynamics in the Dipole-Octupole Pyrochlore Magnet $\mathrm{Ce}_{2} \mathrm{Zr}_{2} \mathrm{O}_{7}$, Phys. Rev. Lett. 122, 187201 (2019).
[19] Y. Li et al., Gapless Quantum Spin Liquid Ground State in the Two-Dimensional Spin-1/2 Triangular Antiferromagnet $\mathrm{YbMgGaO}_{4}$, Sci. Rep. 5, 16419 (2015).

[20] Y. Li, G. Chen, W. Tong, L. Pi, J. Liu, Z. Yang, X. Wang, and Q. Zhang, Rare-Earth Triangular Lattice Spin Liquid: A Single-Crystal Study of $\mathrm{YbMgGaO}_{4}$, Phys. Rev. Lett. 115, 167203 (2015).

[21] D. E. Freedman, T. H. Han, A. Prodi, P. Muller, Q-Z. Huang, Y-S. Chen, S. M. Webb, Y.S. Lee, T. M. Mcqueen, and D. G. Nocera, Site Specifc X-Ray Anomalous Dispersion of the Geometrically Frustrated Kagomé Magnet, Herbertsmithite, $\mathrm{ZnCu}_{3}(\mathrm{OH})_{6} \mathrm{Cl}_{2}$, J. Am. Chem. Soc. 132, 161851 (2010).

[22] Z. Ma, J. Wang, Z. Y. Dong, J. Zhang, S. Li, S. H. Zheng, Y. Yu, W. Wang, L. Che, K. Ran et al., Spin-Glass Ground State in a Triangular-Lattice Compound $\mathrm{YbZnGaO}_{4}$, Phys. Rev. Lett. 120, 087201 (2018).

[23] Z. Zhu, P. A. Maksimov, S. R. White, and A. L. Chernyshev, Disorder-Induced Mimicry of a Spin Liquid in $\mathrm{YbMgGaO}_{4}$, Phys. Rev. Lett. 119, 157201 (2017).

[24] I. Kimchi, A. Nahum, and T. Senthil, Valence Bonds in Random Quantum Magnets: Theory and Application to $\mathrm{YbMgGaO}_{4}$, Phys. Rev. X 8, 031028 (2018).

[25] J. G. Rau and M. J. P. Gingras, Frustration and Anisotropic Exchange in Ytterbium Magnets with Edge-Shared Octahedra, Phys. Rev. B 98, 054408 (2018).

[26] P. A. Maksimov, Z. Zhu, S. R. White, and A. L. Chernyshev, Anisotropic-Exchange Magnets on a Triangular Lattice: Spin Waves, Accidental Degeneracies, and Dual Spin Liquids, Phys. Rev. X 9, 021017 (2019).

[27] W. Liu, Z. Zhang, J. Ji, Y. Liu, J. Li, X. Wang, H. Lei, G. Chen, and Q. Zhang, Rare-Earth Chalcogenides: A Large Family of Triangular Lattice Spin Liquid Candidates, Chin. Phys. Lett. 35, 117501 (2018).

[28] M. Baenitz et al., $\mathrm{NaYbS}_{2}$ : A Planar Spin-1/2 TriangularLattice Magnet and Putative Spin Liquid, Phys. Rev. B 98 , 220409(R) (2018).

[29] K. M. Ranjith et al., Field-Induced Instability of the Quantum Spin Liquid Ground State in the $J_{\mathrm{eff}}=1 / 2$ TriangularLattice Compound $\mathrm{NaYbO}_{2}$, Phys. Rev. B 99, 180401(R) (2019).

[30] Z. Zhang et al., Crystalline Electric-Field Excitations in Quantum Spin Liquids Candidate $\mathrm{NaYbSe}_{2}$, Phys. Rev. B 103, 035144 (2021).

[31] J. Sichelschmidt, P. Schlender, B. Schmidt, M. Baenitz, and T. Doert, Electron Spin Resonance on the Spin-1/2 Triangular Magnet $\mathrm{NaYbS}_{2}$, J. Phys. Condens. Matter 31, 205601 (2019).

[32] Z. Zangeneh, S. Avdoshenko, J. van den Brink, and L. Hozoi, Single-Site Magnetic Anisotropy Governed by Interlayer Cation Charge Imbalance in Triangular-Lattice A $\mathrm{YbX}_{2}$, Phys. Rev. B 100, 174436 (2019).

[33] J. Sichelschmidt, B. Schmidt, P. Schlender, S. Khim, T. Doert, and M. Baenitz, Effective Spin-1/2 Moments on a $\mathrm{Yb}^{3+}$ Triangular Lattice: An ESR Study, J. Phys. Soc. Jpn. Conf. Proc. 30, 011096 (2020).

[34] M. M. Bordelon et al., Field-Tunable Quantum Disordered Ground State in the Triangular-Lattice Antiferromagnet $\mathrm{NaYbO}_{2}$, Nat. Phys. 15, 1058 (2019). 
[35] L. Ding et al., Gapless Spin-Liquid State in the Structurally Disorder-Free Triangular Antiferromagnet $\mathrm{NaYbO}_{2}$, Phys. Rev. B 100, 144432 (2019).

[36] M. M. Bordelon, C. Liu, L. Posthuma, P. M. Sarte, N. P. Butch, D. M. Pajerowski, A. Banerjee, L. Balents, and S. D. Wilson, Spin Excitations in the Frustrated Triangular Lattice Antiferromagnet $\mathrm{NaYbO}_{2}$, Phys. Rev. B 101, 224427 (2020).

[37] J. Guo, X. Zhao, S. Ohira-Kawamura, L. Ling, J. Wang, L. He, K. Nakajima, B. Li, and Z. Zhang, Magnetic-Field and Composition Tuned Antiferromagnetic Instability in the Quantum Spin-Liquid Candidate $\mathrm{NaYbO}_{2}$, Phys. Rev. Mater. 4, 064410 (2020).

[38] See Supplemental Material at http://link.aps.org/ supplemental/10.1103/PhysRevX.11.021044 for details, which includes Refs. [39-42].

[39] S. S. Lee, P. A. Lee, and T. Senthil, Amperean Pairing Instability in the U(1) Spin Liquid State with Fermi Surface and Application to $\kappa-(\mathrm{BEDT}-\mathrm{TTF})_{2} \mathrm{Cu}_{2}(\mathrm{CN})_{3}$, Phys. Rev. Lett. 98, 067006 (2007).

[40] Y. Li, D. Adroja, R. I. Bewley, D. Voneshen, A. A. Tsirlin, P. Gegenwart, and Q. Zhang, Crystalline Electric-Field Randomness in the Triangular Lattice Spin-Liquid $\mathrm{YbMgGaO}_{4}$, Phys. Rev. Lett. 118, 107202 (2017).

[41] G. Bastien et al., Long-Range Magnetic Order in the $\tilde{S}=$ 1/2 Triangular Lattice Antiferromagnet $\mathrm{KCeS}_{2}$, SciPost Phys. 9, 041 (2020).

[42] S. Yamashita, Y. Nakazawa, M. Oguni, Y. Oshima, H. Nojiri, Y. Shimizu, K. Miyagawa, and K. Kanoda, Thermodynamic Properties of a Spin-1/2 Spin-Liquid State in a $\kappa$ Type Organic Salt, Nat. Phys. 4, 459 (2008).

[43] Y. D. Li, Y. M. Lu, and G. Chen, Spinon Fermi Surface U(1) Spin Liquid in the Spin-Orbit-Coupled Triangular-Lattice Mott Insulator $\mathrm{YbMgGaO}_{4}$, Phys. Rev. B 96, 054445 (2017).

[44] K. M. Ranjith et al., Anisotropic Field-Induced Ordering in the Triangular-Lattice Quantum Spin Liquid $\mathrm{NaYbSe}_{2}$, Phys. Rev. B 100, 224417(R) (2019).

[45] J. Xing, L. D. Sanjeewa, J. Kim, G. R. Stewart, A. Podlesnyak, and A. S. Sefat, Field-Induced Magnetic Transition and Spin Fuctuations in the Quantum Spin-Liquid Candidate $\mathrm{Cs} \mathrm{YbSe}_{2}$, Phys. Rev. B 100, 220407(R) (2019).
[46] Y. Li and G. Chen, Detecting Spin Fractionalization in a Spinon Fermi Surface Spin Liquid, Phys. Rev. B 96, 075105 (2017).

[47] A. M. Polyakov, Quark Confinement and Topology of Gauge Theories, Nucl. Phys. B120, 429 (1977).

[48] S. S. Lee, Stability of the U(1) Spin Liquid with a Spinon Fermi Surface in 2+1 Dimensions, Phys. Rev. B 78, 085129 (2008).

[49] Y. Jia et al., Mott Transition and Superconductivity in Quantum Spin Liquid Candidate $\mathrm{NaYbSe}_{2}$, Chin. Phys. Lett. 37, 097404 (2020).

[50] Z. Zhang et al., Pressure Induced Metallization and Possible Unconventional Superconductivity in Spin Liquid $\mathrm{NaYbSe}_{2}$, arXiv:2003.11479.

[51] J. Rodríguez-Carvajal, Recent Advances in Magnetic Structure Determination by Neutron Powder Diffraction, Physica (Amsterdam) 192B, 55 (1993).

[52] G. Ehlers, A. A. Podlesnyak, J. L. Niedziela, E. B. Iverson, and P. E. Sokol, The New Cold Neutron Chopper Spectrometer at the Spallation Neutron Source: Design and Performance, Rev. Sci. Instrum. 82, 085108 (2011).

[53] D. L. Abernathy, M. B. Stone, M. J. Loguillo, M. S. Lucas, O. Delaire, X. Tang, J. . Y. Lin, and B. Fultz, Design and Operation of the Wide Angular-Range Chopper Spectrometer ARCS at the Spallation Neutron Source, Rev. Sci. Instrum. 83, 015114 (2012).

[54] R. I. Bewley, J. W. Taylor, and S. M. Bennington. LET, A Cold Neutron Multi-Disk Chopper Spectrometer at ISIS, Nucl. Instrum. Methods Phys. Res., Sect. A 637, 128 (2011).

[55] X. Lu et al., Fractionalized Magnetic Excitations in a Quantum Spin Liquid Candidate $\mathrm{NaYbSe}_{2}$, STFC ISIS Neutron and Muon Source, https://doi.org/10.5286/ISIS.E.RB1920512.

[56] O. Arnold et al., Mantid-Data Analysis and Visualization Package for Neutron Scattering and $\mu \mathrm{SR}$ Experiments, Nucl. Instrum. Methods Phys. Res., Sect. A 764, 156 (2014).

[57] R. A. Ewings, A. Buts, M. D. Le, J. van Duijn, I. Bustinduy, and T. G. Perring, HORACE: Software for the Analysis of Data from Single Crystal Spectroscopy Experiments at Time-of-Flight Neutron Instruments, Nucl. Instrum. Methods Phys. Res., Sect. A 834, 132 (2016). 